\title{
Transurethral deroofing of midline prostatic cyst for subfertile men
}

\author{
Erik B.Cornel ${ }^{1}$, Gert R.Dohle ${ }^{2}$ and \\ Eric J.H.Meuleman ${ }^{1.3}$ \\ ${ }^{1}$ Department of Urology Academic Hospital Nijmegen, \\ P.O. Box 9101, 6500 HB Nijmegen and ${ }^{2}$ Department of Urology \\ Academic Hospital Rotterdam, P.O. Box 2040, 3000 CA \\ Rotterdam, The Netherlands \\ ${ }^{3}$ To whom correspondence should be addressed \\ E-mail: E.Meuleman@uro.azn.nl
}

We evaluated the efficacy of transurethral deroofing of a midline prostatic cyst in subfertile men with one or more of the following semen abnormalities: decreased ejaculatory volume, decreased sperm motility and oligo- or azoospermia. Results from treatment of a series of 11 subfertile men with a midline prostatic cyst by transurethral deroofing of the cyst are presented. Five patients showed an improvement of seminal volume. Only one patient demonstrated an improvement of sperm count. Sperm motility was not influenced. No relationship was found between positive outcome following operation and either size of the cyst or dilatation of the seminal vesicles. Spontaneous pregnancies did not occur after transurethral deroofing of the midline prostatic cyst. In conclusion, our study suggests a poor efficacy of transurethral deroofing of a midline prostatic cyst in subfertile men with the above mentioned semen abnormalities.

Key words: male subfertility/prostatic cyst/transurethral deroofing

\section{Introduction}

In the last decade refinement of assisted reproductive techniques have gained importance in the treatment of male subfertility. Due to the success of assisted reproductive techniques, irrespective of the aetiology of male subfertility, appropriate evaluation is often bypassed although several causes can be effectively treated. In almost half of the infertile couples, male subfertility, defined as the failure to induce a pregnancy within one year of regular unprotected intercourse (Meuleman, 1998), is present (Mosher and Pratt, 1991). It is therefore of importance to identify those subfertile males for whom treatment options are available before embarking on expensive and potentially hazardous assisted reproductive techniques.

Ejaculatory duct obstruction (EDO) is regarded as one of the treatable causes of male subfertility. EDO is found in 7$14 \%$ of the subfertile male population and can be congenital or acquired (Goldwasser et al., 1985; Pryor and Hendry, 1991).
Normal hormone status and one or more of the following distinct semen abnormalities such as low or absent fructose, decreased sperm motility, decreased ejaculatory volume and oligo- or azoospermia are suggestive for total or partial EDO. The diagnosis of EDO can be confirmed directly by vasography (Banner and Hassler, 1978) or indirectly by transrectal ultrasonography (TRUS) (Jarow, 1993). Due to its non-invasive nature TRUS is currently the diagnostic method of choice.

Theoretically, a midline prostatic cyst can be one of the causes of EDO. The cyst, localized in the central zone of the prostate, can compress or displace the ejaculatory ducts to the lateral side (Fisch, 1992). It has been our policy to offer transurethral deroofing of the prostatic cyst to subfertile men with one or more of the following semen abnormalities: decreased seminal volume, decreased motility, oligozoospermia or azoospermia, in order to improve semen quantity and quality and pregnancy rate. In this retrospective study we evaluated the efficacy of this treatment.

\section{Materials and methods}

Since 199611 patients presented in our departments with subfertility were diagnosed with a median prostatic cyst. Age ranged from 2539 years, mean 34 years. There were no urological complaints. Infertility was primary in 10 patients and one patient developed azoospermia after fathering one son. At physical examination all patients had normal sized testes $(15-25 \mathrm{ml})$, bilateral palpable vas deferens and absence of varicoceles. Follicle stimulating hormone and testosterone were measured and always normal. Semen analyses were performed at least twice before operation. Patients were asked to abstain from sexual activity for 2 to 3 days before semen collection. Semen evaluation was performed at the university-based andrology laboratory and included ejaculate volume, seminal plasma $\mathrm{pH}$, sperm concentration, percentage of sperm motility and sperm viability. TRUS of the prostate, performed if EDO was suspected, demonstrated the presence of a midline prostatic cyst and, if present, concurrent dilatation of seminal vesicles or ejaculatory duct.

The diagnosis of subfertility based on a median prostatic cyst was based on semen analysis with one or more of the following findings in addition to normal physical examination and normal serum FSH and testosterone: (i) low ejaculate volume $(<2 \mathrm{ml})$ azoospermia; (ii) low or normal ejaculate volume with a sperm concentration $<20 \times 10^{6} \%$ $\mathrm{ml}$; or (iii) low or normal ejaculate volume with sperm motility $<30 \%$ (Kim et al., 1997).

Transurethral incision of the midline cyst was performed under local anaesthesia with the patient in the lithotomy position. The roof of the cyst was incised, under TRUS guidance and under direct vision through a Collins hook (Karl Storz GmbH and Co., Tutthingen, Germany). Generally, the prostatic floor had to be incised between the bladder neck and the verumontanum that resulted in complete marsupialization of the cyst. Minimal coagulation was used. A Ch 16 


\begin{tabular}{|c|c|c|c|c|c|c|c|}
\hline \multicolumn{2}{|c|}{ Patient no. } & \multirow{2}{*}{$\begin{array}{l}\text { Obstruction } \\
\text { signs }\end{array}$} & \multirow{2}{*}{$\begin{array}{l}\begin{array}{l}\text { Semen } \\
\text { volume } \\
(\mathrm{ml})\end{array} \\
0.6\end{array}$} & \multirow{2}{*}{$\begin{array}{l}\begin{array}{l}\text { Sperm } \\
\text { concentration } \\
\left(\times 10^{6} / \mathrm{ml}\right)\end{array} \\
0\end{array}$} & \multirow{2}{*}{$\begin{array}{l}\% \text { motility } \\
-\end{array}$} & \multirow{2}{*}{$\begin{array}{l}\text { Motility } \\
\text { quality }\end{array}$} & \multirow{2}{*}{$\begin{array}{l}\text { \% abnormal } \\
-\end{array}$} \\
\hline 1 & Pre-op & & & & & & \\
\hline & Post-op & & 0.9 & 0 & - & - & - \\
\hline \multirow[t]{2}{*}{2} & Pre-op & Yes & 1.7 & 3.3 & 5 & 3 & 71 \\
\hline & Post-op & & 1.5 & 2.6 & 35 & 3 & 76 \\
\hline \multirow[t]{2}{*}{3} & Pre-op & No & 1.6 & $<0.1$ & - & 1 & ND \\
\hline & Post-op & & 3.0 & $<0.1$ & - & 1 & ND \\
\hline \multirow[t]{2}{*}{4} & Pre-op & Yes & 0.6 & 30 & - & 1 & - \\
\hline & Post-op & & 0.7 & 1.0 & - & 1 & - \\
\hline \multirow[t]{2}{*}{5} & Pre-op & Yes & 0.5 & 58 & 15 & 3 & 45 \\
\hline & Post-op & & 1.7 & 14 & 50 & 3 & 55 \\
\hline \multirow[t]{2}{*}{6} & Pre-op & No & 0.8 & 0 & - & 1 & - \\
\hline & Post-op & & 0.7 & 25 later 0 & 45 later - & 4 later 1 & 58 later - \\
\hline \multirow[t]{2}{*}{7} & Pre-op & Yes & 0.3 & 0.2 & - & 1 & - \\
\hline & Post-op & & 0.3 & 0.4 & - & 1 & 1 \\
\hline \multirow[t]{2}{*}{8} & Pre-op & no & 0.3 & 15 & - & 1 & - \\
\hline & Post-op & & 3.4 & 15 & - & 1 & - \\
\hline \multirow[t]{2}{*}{9} & Pre-op & no & 0.3 & 0.1 & - & 1 & - \\
\hline & Post-op & & 0.3 & 0.1 & - & 1 & - \\
\hline \multirow[t]{2}{*}{10} & Pre-op & No & 0.5 & 5.4 & 7 & 3 & 94 \\
\hline & Post-op & & 0.9 & 14 & 13 & 3 & 94 \\
\hline \multirow[t]{2}{*}{11} & Pre-op & No & 0.1 & 45 & 38 & 4 & 84 \\
\hline & Post-op & & 1.2 & 46 & 46 & 4 & 90 \\
\hline
\end{tabular}

Motility quality: (1) none, (2) bad, (3) moderate, (4) quite good, (5) good, (6) excellent.

Pre-op/post-op $=$ pre- and post-operative.

$\mathrm{ND}=$ not determined because amount too low.

transurethral catheter (Bardex I.C.; Bard Benelux n.v., Olen, Belgium) was introduced in the bladder and removed $24 \mathrm{~h}$ post-operatively. Patients were discharged the day after the procedure. There were no complications after transurethral incision of the midline cyst and this procedure was well tolerated by all patients. Semen analyses were obtained 4-12 weeks postoperatively.

\section{Results}

Table I summarizes pre- and post-operative seminal parameters of all patients. Pre-operative seminal parameters corresponded to the mean values of at least two semen analyses performed within 6 months before transurethral incision of the midline prostatic cyst. Post-operative seminal parameters corresponded to the mean values of at least two semen analyses performed at the latest follow-up.

\section{Effect of transurethral incision of the midline prostatic cyst on sperm volume}

Patients 3, 5, 8, 10 and 11 showed improvement of seminal volume after the procedure (Table I). No relationship was found between the positive effect of the incision and the size of the cyst or dilatation of the seminal vesicles.

\section{Effect of transurethral incision of the midline prostatic cyst on sperm quality}

Sperm concentration did not change in eight patients, decreased in patient no. 4 and increased only in patient no. 10 (Table I). Additionally the percentage of motile spermatozoa and the quality of motility did not change after surgery. A remarkable, but transient, improvement in sperm quality was seen in one patient (no. 6). In the first 3 months following the transurethral incision of the cyst, semen analysis showed dramatic sperm quantity improvement, but eventually the patient developed azoospermia again.

\section{Effect of transurethral incision of the midline prostatic cyst on pregnancy rate}

No spontaneous pregnancies occurred following transurethral incision.

\section{Discussion}

The widespread utilization of TRUS to diagnose prostate abnormalities and as a diagnostic tool in the evaluation of low ejaculate volume, azoospermia or oligozoospermia in subfertile men has resulted in more frequent detection of midline prostatic cysts (Kim et al., 1997). These cysts can be of utricular or Müllerian duct origin (Golubuff et al., 1995). Utricular cysts are of endodermal origin, contain no spermatozoa and are located near the verumontanum whereas Müllerian cysts are of mesodermal origin, contain spermatozoa and are located more posterior and nearer the prostate base. The ejaculatory ducts enter the prostate obliquely posteriorly at its base, course medially and anteriorly through the glandular tissue of the prostate and end in the prostatic urethra at the verumontanum (Golubuff et al., 1995). Goluboff et al. (1995) demonstrated that the ejaculatory ducts run in an almost straight course from the prostatic base to the verumontanum (Golubuff et al., 1995) 
and it is therefore believed that a midline prostatic cyst can be one of the causes of ejaculatory duct obstruction by lateral compression of the ejaculatory ducts (Fisch, 1992).

Historically, ejaculatory duct obstruction was diagnosed by vasography (Pryor and Hendry, 1991; Jarow, 1993). A complete block in the ejaculatory ducts is conclusive for the diagnosis of total obstruction. The main drawback, however, is the invasiveness of the procedure, and the subsequent risk of iatrogenic occlusion. In contrast, TRUS is readily available, inexpensive and non-invasive. The ultrasonographic diagnosis of EDO is based upon dilation of seminal vesicles or abnormalities such as midline prostatic cysts or calcifications in the region of the ejaculatory ducts. Unfortunately not all patients with EDO have dilated seminal vesicles and, conversely, not all patients with dilated seminal vesicles have EDO (Littup et al., 1988). Moreover, the functional implication of a midline prostatic cyst or prostatic calcifications cannot be determined by TRUS (Jarow, 1996a). It provides only circumstantial evidence for obstruction. Nevertheless, at present TRUS has replaced vasography as imaging modality for suspected ejaculatory duct obstruction.

Several treatments for EDO caused by midline prostatic cysts, e.g. transurethral resection of the ejaculatory ducts (TURED), transrectal aspiration together with sclerotherapy, transurethral marsupialization and open surgery of the midline prostatic cyst have been described (Ritchey et al., 1988; Fisch, 1992; Stricker et al., 1993; Jarow, 1996b).

Currently, the standard treatment has become TURED (Fisch, 1992; Mecham et al., 1993). The overall success rate of TURED has been quite good (Jarow, 1996b). In the literature more than 100 patients have been described as having had this procedure for subfertility with an improvement of semen parameters seen in 50\%. Spontaneous pregnancies occurred in $25 \%$ of the cases (Jarow, 1996b; Netto et al., 1988). Hendry and Pryor (1992) performed a transurethral incision of a Müllerian cyst in 21 patients, of whom 10 (48\%) experienced an undisclosed improvement in semen quality and eight (38\%) partners conceived (Hendry and Pryor, 1992). Dik et al. (1996) described in their series of transurethral marsupialization of medial prostatic cyst in patients with prostatitis-like symptoms, three patients with infertility of whom two demonstrated significant improvement in semen quantity and quality (Dik et al., 1996). Moreover, eight of the 10 patients with prostatitislike symptoms who had pre-operatively small volume ejaculation without infertility demonstrated improvement in semen volume after the transurethral marsupialization (Dik et al., 1996). These results clearly advocate a functional relationship between midline prostatic cysts and low semen volume. Our poor results of transurethral incision of midline prostatic cyst for infertility are therefore surprising. Only five (46\%) patients demonstrated an improvement in seminal volume and in one patient improvement of sperm concentration was seen. Sperm motility was not affected in any patient. In our series spontaneous pregnancies did not occur after transurethral deroofing of the midline prostatic cyst.

The diagnosis of subfertility caused by obstruction due to midline prostatic cyst was based on history, physical examination, semen analyses and TRUS. Since vasography was not used in the diagnostic process, a functional relationship between the midline prostatic cyst and obstruction was not established, which may explain our poor results. Functional investigation, i.e. seminal vesiculography or vasography, may still be mandatory to prove the existence of an obstruction and to prove its removal afterwards. Secondly, the midline prostatic cyst was marsupialized and the cyst walls were not resected. The edges may heal together once again thus allowing the obstruction to return. Theoretically, a midline prostatic cyst can cause direct obstruction of the ejaculatory ducts by compression, but secondary fibrosis/stenosis of the ejaculatory ducts might occur on different levels between the ejaculatory duct orifice in the urethra and the epididymal junction. Although marsupialization of the midline cyst might solve the direct obstructive factor, the secondary obstruction might still be present. Digital massage of the seminal vesicles, possibly prior to the operation and injection with a coloured dye, could therefore be helpful to check patency of the ejaculatory ducts following marsupialization of the cyst.

Finally, an improved patient selection might influence pregnancy outcome. Patients with subfertility based only on low semen volume might benefit from improvement of seminal volume because the concomitant increase of $\mathrm{pH}$ of the seminal fluid may protect the spermatozoa against the harmful influence of the acid vaginal mucous.

In conclusion, our study suggests a poor efficacy of transurethral deroofing of a midline prostatic cyst alone in subfertile males with low semen volume, oligozoospermia or azoospermia. To improve the results, selective vasography or vesicography prior to an incision to confirm obstruction may be useful despite the potential harmful effects. Moreover, marsupialization of the cyst in combination with a resection of the wall of the cyst might improve the results. Only time will tell if these adjustments will improve the results, in terms of pregnancy rates, of transurethral deroofing of a midline prostatic cyst in subfertile men with low semen volume and/or azooor oligozoospermia.

\section{References}

Banner, M.P. and Hassler, R. (1978) The normal vesiculogram. Radiology, 128, 339-344.

Dik, P., Lock, T.M.W.T., Schrier, B.P. et al. (1996) Transurethral marsupialization of a medial prostatic cyst in patients with prostatitis-like symptoms. J. Urol., 155, 1301-1304.

Fisch, H. (1992) Transurethral resection of the ejaculatory ducts. Curr. Surg. Tech. Urol., 5, 2-7.

Goldwasser, B.Z., Weinerth, J.L. and Carson, C.C. III (1985) Ejaculatory duct obstruction: the case for aggressive diagnosis and treatment. J. Urol., 134, 964-966.

Goluboff, E.T., Stifelman, M.D. and Fisch, H. (1995) Ejaculatory duct obstruct in infertile men. Urology, 45, 925-931.

Jarow, J.P. (1993) Transrectal ultrasonography in the infertile men. Fertil. Steril., 60, 1035-1039.

Jarow, J.P. (1996a) Transrectal ultrasonography in the diagnosis and management of ejaculatory duct obstruction. J. Androl., 17, 467-472.

Jarow, J.P. (1996b) Diagnosis and management of ejaculatory duct obstruction. Techniques in Urology, 2, 79-85.

Kim, E.D., Onel, E. and Honing, S.C. (1997) The prevalence of cystic abnormalities of the prostate involving the ejaculatory ducts as detected by transrectal ultrasound. Int. Urol. Nephrol., 29, 647-652.

Hendry, W.F. and Pryor, J.P. (1992) Müllerian duct (Prostatic utricle) cyst: Diagnosis and treatment in subfertile males. Br. J. of Urol., 69, 79-82. 
Littup, P.J., Lee, F. and McLeary, R.D. (1988) Transrectal US of the seminal vesicles and ejaculatory ducts: clinical correlation. Radiology, 168, 625-628.

Mecham, R.B., Hellerstein, D.K. and Lipshultz, L.I. (1993) Evaluation and treatment of ejaculatory duct obstruction in the infertile male. Fertil. Steril., 59, 393-397.

Meuleman, E.J.H. (1998) Male infertility. Eur. Urol., 34, see Curric. urol., 5, $1-8$.

Mosher, W.D. and Pratt, W.F. (1991) Fecundity and infertility in the United States: incidence and trends. Fertil. Steril., 56, 192-193.

Netto, N.R., Esteves, S.C. and Neves, P.A. (1988) Transurethral resection of partially obstructed ejaculatory ducts: seminal parameters and pregnancy outcome according to the etiology of obstruction. J. Urol., 159, 2048-2053.

Pryor, J.P. and Hendry, W.F. (1991) Ejaculatory duct obstruction in subfertile males: analysis of 87 patients. Fertil. Steril., 56, 725-730.

Ritchey, M.L., Benson, R.C., Kramer, S.A. et al. (1988) Management of müllerian duct remnants in the male patient. J. Urol., 140, 795-799.

Stricker, H.J., Kunin, J.R., Faeber, G.J. et al. (1993) Congenital prostatic cyst causing ejaculatory duct obstruction: management by transrectal aspiration. J. Urol., 149, 1141-1143.

Received on March 4, 1999; accepted on June 8, 1999 